\title{
Engineered fungal polyketide biosynthesis in Pichia pastoris: a potential excellent host for polyketide production
}

\author{
Limei Gao, Menghao Cai ${ }^{*}$ Wei Shen, Siwei Xiao, Xiangshan Zhou and Yuanxing Zhang
}

\begin{abstract}
Background: Polyketides are one of the most important classes of secondary metabolites and usually make good drugs. Currently, heterologous production of fungal polyketides for developing a high potential industrial application system with high production capacity and pharmacutical feasibility was still at its infancy. Pichia pastoris is a highly successful system for the high production of a variety of heterologous proteins. In this work, we aim to develop a P. pastoris based in vivo fungal polyketide production system for first time and evaluate its feasibility for future industrial application.
\end{abstract}

Results: A recombinant P. pastoris GS115-NpgA-ATX with Aspergillus nidulans phosphopantetheinyl transferase (PPtase) gene npgA and Aspergillus terrus 6-methylsalicylic acid (6-MSA) synthase (6-MSAS) gene atX was constructed. A specific compound was isolated and idenified as 6-MSA by HPLC, LC-MS and NMR. Transcription of both genes were detected. In 5-L bioreactor, the GS115-NpgA-ATX grew well and produced 6-MSA quickly until reached a high value of $2.2 \mathrm{~g} / \mathrm{L}$ by methanol induction for 20 hours. Thereafter, the cells turned to death ascribing to high concentration of antimicrobial 6-MSA. The distribution of 6-MSA changed that during early and late induction phase it existed more in supernatant while during intermediate stage it mainly located intracellular. Different from 6-MSA production strain, recombinant M. purpureus pksCT expression strains for citrinin intermediate production, no matter PksCT located in cytoplasm or in peroxisomes, did not produce any specfic compound. However, both npgA and pksCT transcripted effectively in cells and western blot analysis proved the expression of PPtase. Then the PPTase was expressed and purified, marked by fluorescent probes, and reacted with purified ACP domain and its mutant ACPm of PksCT. Fluoresence was only observed in ACP but not ACPm, indicating that the PPTase worked well with ACP to make it bioactive holo-ACP. Thus, some other factors may affect polyketide synthesis that include activities of the individual catalytic domains and release of the product from the synthase of PksCT.

Conclusions: An efficient P. pastoris expression system of fungal polyketides was successfully constructed. It produced a high production of 6-MSA and holds potential for future industrial application of 6-MSA and other fungal polyketides.

Keywords: Polyketide, Pichia pastoris, 6-MSA, 6-MSAS, Heterologous expression, Fermentation

\footnotetext{
* Correspondence: cmh022199@ecust.edu.cn

State Key Laboratory of Bioreactor Engineering, East China University of

Science and Technology, Shanghai 200237, China
} 


\section{Background}

Polyketides are one of the most important classes of secondary metabolites. They occur in bacteria, fungi, plants, marine organisms, etc., showing flexible structural diversity and a variety of bioactivities [1]. Polyketides and their derivatives make good drugs. As reported by Weissman and Leadlay in 2005, polyketide-derived pharmaceuticals comprise $20 \%$ of the top-selling drugs, with combined worldwide revenues of over UK $£ 10$ billion per year [2]. Many important drugs have suffered or will face expiration of the key patents, which is likely to lead to explosive production growth of these compounds [3].

During the last two decades, the biosynthetic mechanism of polyketides catalyzed by polyketide synthase (PKS) have been well interpreted despite that some highly controlled synthetic processes still remained unknown. As is known, fungal PKSs belong to iterative type I PKS that contains an obligatory set of ketosynthase (KS), acyltransferase (AT) and acyl carrier protein (ACP) domains, which is closely related to fatty acid biosynthesis [1].The ACP domains carry the malonyl extender units and transiently link the growing acyl chain. Before functioning, the apo-ACP requires post-translational modification to be holo-ACP through the addition of phosphopantetheine (PP) derived from coenzyme A (CoA) to a conserved serine residue catalyzed by phosphopantetheinyl transferase (PPtase). The Claisen condensation is catalyzed by the KS while the AT transfers acyl groups from CoA onto the KS and ACP domains. The resulted $\beta$-ketothioester could be further processed by $\beta$-ketoacyl reductase (KR), dehydratase $(\mathrm{DH})$ and/or enoyl reductase (ER) to form an ester with different degree of saturation. Fungal PKS can own another intrinsic domain C-methyl transferase (CMeT) that additionally methylated the chain using a methyl group from S-adenosylmethionine (SAM). Once the polyketide chain has finished, a thioesterase (TE) hydrolyses the thioester and releases the carbon chain from the enzyme, although it is not found in every PKS. The recycling mechanism of fungal type I PKS makes the biosynthesis process efficiently with relatively small genes.

Due to the broad bioactivity and well known synthesis mechanism of polyketides their production have attracted much interest in the field of metabolic engineering. To present, several hosts, i.e., E.coli, Saccharomyces cerevisiae, Streptomyces coelicolor, Aspergillus nidulans, Aspergillus oryzae, etc., have already been accomplished for polyketides biosynthesis [4]. An reconstruction of a complete functional fungal biosynthetic multigene cluster even succeeded in A. oryzae [5]. However, these studies mainly focused on identifying the functions of PKSs, performing combinational biochemistry for new compound biosynthesis, or testing the feasibility of the expression system, while the work for developing a high potential industrial application system with high production capacity of pharmaceutical compound was still at its infancy. It is believed that, combinational biochemistry would play an important role in future drug development and explore a highly efficient heterologous production system turns to be critical.

As is known, Pichia pastoris is well used as a highly successful system for the production of a variety of heterologous proteins in both laboratory and industry [6,7]. For $P$. pastoris, the simple molecular genetic manipulation and commercially available kit made it conveniently for laboratory and industrial application. It can produce foreign proteins at high levels under control of methanol-inducible strong promoter $A O X 1$, either intracellularly or extracellularly. Moreover, it has the capability of directing proteins into peroxisome and performing many eukaryotic post-translational modifications, such as glycosylation, disulfide bond formation and proteolytic processing. However, till now, there is few reports focused on polyketide synthase (PKS) expression triggering compound biosynthesis in vivo by $P$. pastoris.

The PKS of 6-methylsalicylic acid (6-MSA) synthase (6-MSAS) responsible for 6-MSA biosynthesis was the first fungal PKS gene to be cloned and is also one of the best characterized fungal PKSs [8-10]. It has been commonly used as a model PKS for evaluation of heterologously expression system such as $S$. cerevisiae $[3,11]$, S. coelicolor [12], E. coli [13], tobacco [14]. Mycotoxin citrinin was originally isolated from Penicillium citrinum and now produced by a variety of other fungi [15]. It damages human health and usually accompanies with pigment production in Monascus spp., and gives rise to wide attention. A gene cluster for biosynthesis of mycotoxin citrinin have been reported, in which, a $p k s C T$ plays an important role and probably responsible for its intermediate biosynthesis $[16,17]$. For heterologous production of polyketide in yeast, the post-translational modification of the ACP by phosphopantetheinylation is necessary. As previously reported, this challenge could be solved by the PPTase encoding genes of npgA from $A$. nidulans or sfp from Bacillus subtilis [3,13].

In this work, we dedicated to establish a P. pastoris based polyketide production system. The synthetic pathway of model fungal polyketide 6-methylsalicylic acid encoded by at $X$ from Aspergillus terreus $[10,18]$ and citrinin intermediate encoded by $p k s C T$ from Monascus purpureus were constructed to verify the feasibility of heterologous production of fungal polyketide in P. pastoris. Besides, bioreactor fermentation was also performed to evaluate the polyketide productivity of the recombinant strain.

\section{Results and discussion}

Construction of strain GS115-NpgA, GS115-ATX and GS115-NpgA-ATX

For polyketide biosynthesis, the ACP functions after post-translational modification by phosphopantetheine 
transferase (PPTase), leading to the transfer of $4{ }^{\prime}$ phosphopantetheine from coenzyme $\mathrm{A}(\mathrm{CoA})$ to a conserved serine residue of ACP [1]. In this study, $A$. nidulans npgA (GenBank: AAF12814) encoding the PPTase consisted of 344 amino acids was selected. Strains were constructed following Section Plasmids and strains. Commercial vector pPIC3.5 K (Invitrogen) with AOX1 promoter and selective marker HIS4 was used for plasmid pPIC3.5 K-NpgA construction and then transformed auxotrophic his 4 wild type P. pastoris GS115 by a single homologous recombination event to integrate at HIS4 locus. A positive transformant P. pastoris GS115NpgA was screened and fully identified by PCR. The 6-MSAS containing 1803 amino acids encoded by at $X$ (GenBank: D85860) from $A$. terrus was expressed by $P$. pastoris and purified with Nickel-affinity chromatography. The intron removed $a t X$ was cloned from a gift plasmid pESC-ATX. The vector pPICZ B (Invitrogen) with $A O X 1$ promoter and selective marker of zeocin resistance gene $S h$ ble was used for plasmid pPICZ B-ATX construction and then transformed $P$. pastoris GS115 and recombinant $P$. pastoris GS115-NpgA by single homologous recombination events to integrate at 5'AOX1 region. The positive methanol utilization plus $\left(\mathrm{Mut}^{+}\right.$) transformants GS115-ATX and GS115NpgA-ATX were screened and fully identified by PCR analysis.

\section{Product identification of GS115-NpgA-ATX}

Three strains, GS115, GS115-ATX and GS115-NpgA-ATX, were cultivated under same conditions. After methanol induced expression for $36 \mathrm{~h}$, the products were extracted and analyzed by HPLC analysis. As shown in Figure 1, GS115-NpgA-ATX produced a specfic compound emerged as a sharp peak in HPLC chromatogram at 16.7 min compared with GS115 and GS115-ATX, which had the same retention time as an authentic sample of 6-MSA under this elution condition. To further confirm the structure of the compound, the extracts were purified by TLC (petroleum ether:ethyl acetate $=3: 1(\mathrm{v} / \mathrm{v}), 1 \%$ acetic acid) and extracted and freeze-dried for further EI-MS and NMR analysis. The EI-MS analysis was performed on an Agilent G2577A mass spectrometer, establishing the molecular formulae as $\mathrm{C}_{8} \mathrm{H}_{8} \mathrm{O}_{3}$ for 6-MSA (with the $\mathrm{M}^{+}$ion at $\mathrm{m} / \mathrm{z}=$ 152.0474, 152.0473 calculated) absolutely accorded with the standard 6-MSA (Figure 2A). The freeze-dried sample dissolved in deuterated DMSO for ${ }^{1} \mathrm{HNMR}$ analysis. The results that ${ }^{1} \mathrm{HNMR}(400 \mathrm{MHz}$, DMSO-d6), $\delta \mathrm{H}=6.37(1 \mathrm{H}, \mathrm{m}, \mathrm{H}-3), 6.94(1 \mathrm{H}, \mathrm{m}, \mathrm{H}-4), 6.46(1 \mathrm{H}$, m, H-5), $2.52\left(3 \mathrm{H}, \mathrm{s}, \mathrm{CH}_{3}-6\right)$ conformed with ${ }^{1} \mathrm{HNMR}$ spectrum of standard 6-MSA and further confirmed the compound (Figure 2B). For all three tested strains, only GS115-NpgA-ATX produced 6-MSA but not GS115-
ATX and wild type GS115, proving that 6-MSAS could be well modified by PPtase and it only works for 6-MSA biosynthesis after PPtase modification. Furthermore, transcription analysis of GS115 and GS115-NpgA-ATX were carried out after 24 hours induction. Three pairs of primers, 5AOX1/3AOX1, NpgAF/NpgAR1, BstpF/ AtxR, were used to test gene transcriptions of $A O X 1$, $n p g A$ and $a t X$. The wild type GS115 only generated the correct nucleic acid size of $2.2 \mathrm{~kb}$ for the $A O X 1$, while GS115-NpgA-ATX formed other two desired PCR products (1.3 kb for $n p g A$ and $1.9 \mathrm{~kb}$ for at $X$ ) besides the $A O X 1$ (Figure 2C), indicating that both $n p g A$ and at $X$ transcripted effectively in the recombinant strain GS115-NpgA-ATX.

\section{Fermentation of GS115-NpgA-ATX in 5-L bioreactor}

A 5-L bioreactor fermentation was subsequently conducted to evaluate the productivity of 6-MSA by the recombinant GS115-NpgA-ATX. As shown in Figure 3, a batch phase in BSM medium proceeded until $32 \mathrm{~h}$ when glycerol used up accompanied with dissolved oxygen sharply increase, and reached a wet cell weight (WCW) of $112 \mathrm{~g} / \mathrm{L}$. A fed-batch phase continued with limited feedings of glycerol feeding medium to improve cell growth until $46 \mathrm{~h}$ when a high cell density of $252 \mathrm{~g} / \mathrm{L}$ (WCW) was achieved. Then, methanol induction phase started at $47 \mathrm{~h}$ after glycerol exhaused with derepression of AOX1 promoter. The 6-MSA rapidly synthesized and accumulated. After induction for 20 hours, 6-MSA production reached up to the highest $2.2 \mathrm{~g} / \mathrm{L}$. However, the culture broth turned extremely abnormal and cells turned to death by methylene blue staining analysis. During methanol induction phase, cell growth was highly repressed, which should be ascribed to the antimicrobial activity of 6-MSA [19]. The repression effect of 6-MSA on $P$. pastoris growth was subsequently verified in 5-L bioreactor fermentation with commerially purchased 6-MSA addition with final concentration of $2.05 \mathrm{~g} / \mathrm{L}$ immediately after WCW reached $235 \mathrm{~g} / \mathrm{L}$ (Additional file 1: Figure S1). For GS115-NpgA-ATX, the distribution of 6-MSA changed that during early induction phase and late phase it existed more in supernatant while during intermediate stage it mainly located intracellular. It might attribute to that the increased methanol feeding rate up-regulated the expression rate of 6-MSAS so that enhanced 6-MSA synthesis. The rapidly accumulated 6-MSA could not be efficiently transferred extracellular. However, during the late induction phase, the death of cells may accelarated 6-MSA release to fermentation broth.

Kealey et al. reported a heterogous expression of 6-MSA with a low production of $75 \mathrm{mg} / \mathrm{L}$ in $E$. coli and a high production of $1.7 \mathrm{~g} / \mathrm{L}$ in $S$. cerevisiae with coexpression of Penicillium patulum MSAS and $B$. subtilis SFP [13]. Wattanachaisaereekul et al. obtained a 6-MSA production of $0.2 \mathrm{~g} / \mathrm{L}$ by heterologous expression 


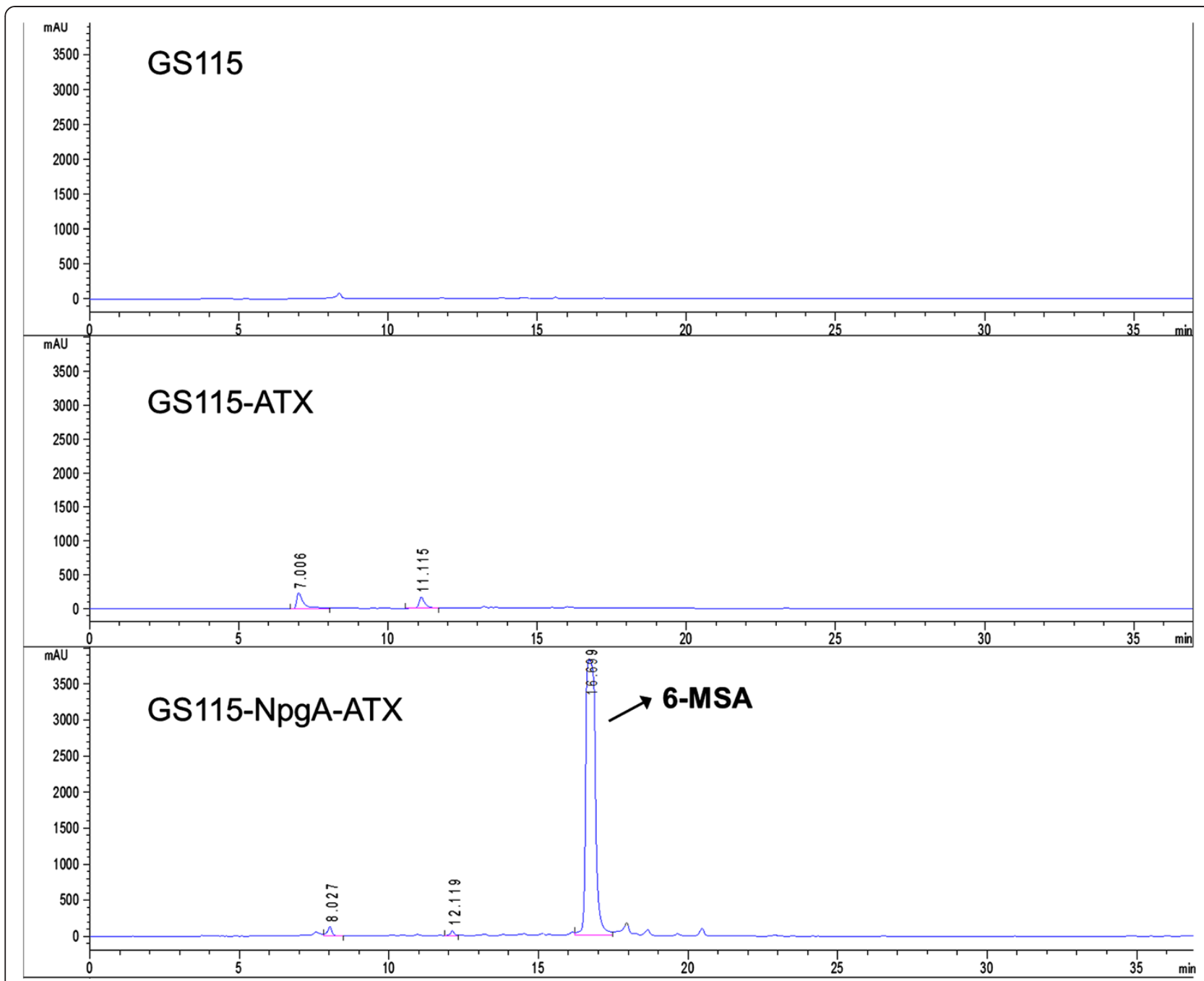

Figure 1 The HPLC chromatogram of organic extracts from fermentation broth of strain GS115, GS115-ATX and GS115-NpgA-ATX.

Samples preparation were described in the Section 6-MSA extraction and identification in Methods.

of A. nidulans NpgA and B. subtilis SFP [3]. They then optimized the expression by swapping promoter $P_{A C C I}$ to a strong, constitutive promoter $P_{T E P 1}$ to increase malonylCoA supply and resulted in an increased 6-MSA production of $0.5 \mathrm{~g} / \mathrm{L}$ [20]. The $6-\mathrm{MSA}$ of $2.2 \mathrm{~g} / \mathrm{L}$ in this case is higher than these published data. It could be further to go by promoting cell density before methanol inducation or developing a fermentation and bioseparation coupling process that releasing 6-MSA from broth to keep cell alive.

\section{Product, transcription and western blot analysis of PksCT expression strains}

The previous study showed that a 56 bp intron presented at 640 to $695 \mathrm{bp}$, flanked by a typical splice site (5'-GT-AG-3') in pksCT, a gene critical for citrinin biosynthesis in $M$. purpureus [21]. The PksCT possessed KS-AT-ACP-MT domains but no TE domain [21]. However, in this study, we found another intron located at
6547 to 6608 bp beside the reported one by RT-PCR. The cDNA of $p k s C T$ by removing two introns were $6507 \mathrm{bp}$, coded for 2169 amino acids, which also contained the intact KS-AT-ACP-MT domains. The expression strains, i.e., GS115-3.5 K, GS115-3.5 K-CT-SKL, GS115-NpgA-CT-SKL, GS115-NpgA-SKL-CT-SKL and GS115-NpgA-CT, were then constructed as that described in Methods and the integration mechanism were the same to construction of atX recombinant strains. However, different from 6-MSAS expression strains, none of them produced specific compound comparing with the control GS115 by HPLC-MS. The strains expressing $p k s C T$ that only removed the front $56 \mathrm{bp}$ intron were also constructed but were still nothing different (data not shown).

Transcription of GS115-3.5 K-CT-SKL, GS115-NpgA-CT and GS115-NpgA-SKL-CT-SKL were then analyzed after 24 hours induction. Two pairs of primers, NpgA-HIS6-F/ 

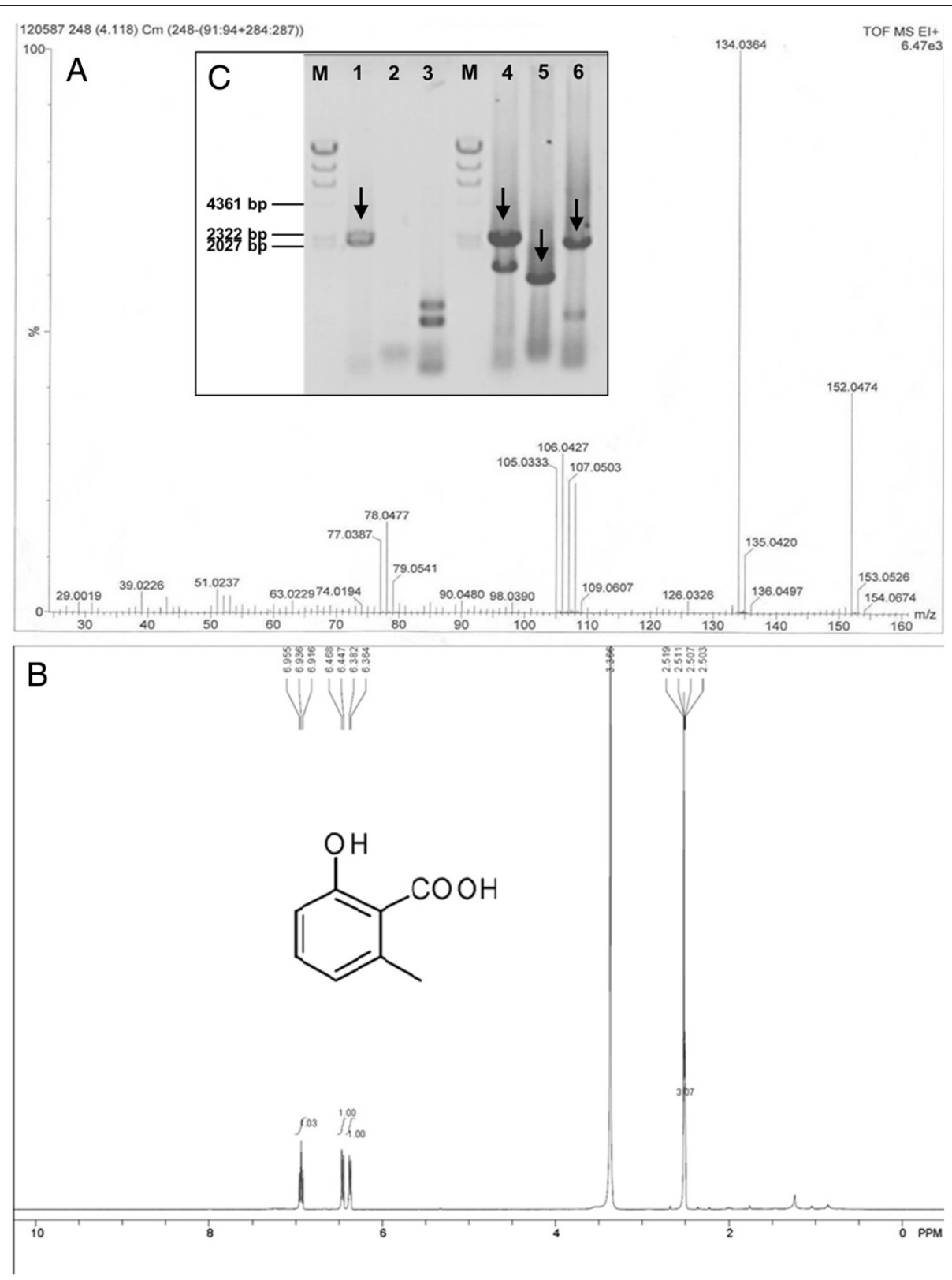

Figure 2 The El-MS identification of product 6-MSA by GS115-NpgA-ATX and transcription analysis of $n p g A$ and atX. (A) El-MS analysis of extract from GS115-NpgA-ATX. m/z, mass-to-charge ratio; (B) The ${ }^{1}$ HNMR analysis of extract from GS115-NpgA-ATX. Samples preparation were in the Section 6-MSA extraction and identification in Methods; (C) Lane 1-3: PCR using CDNA of wild type GS115 and primers 5AOX1/3AOX1, NpgA-F/R1 and BstpF/AtxR, respectively; Lane 4-6: PCR using GS115-NpgA-ATX cDNA and primers 5AOX1/3AOX1, NpgAF/ NpgAR1 and BstpF/AtxR, respectively; M: DNA marker Hind III. Gene transcription induced by $0.5 \%$ methanol for $24 \mathrm{~h}$.

NpgA-HIS6-R and 1977 F/4088R were used to test gene transcriptions of $n p g A$ and $p k s C T$. GS115-3.5 K-CT-SKL acted no npgA transcription but GS115-NpgA-CT and GS115-NpgA-SKL-CT-SKL did (1.1 kb) (Figure 4A). As for $p k s C T$, all three strains transcripted normally $(2.1 \mathrm{~kb})$ (Figure 4B). A western blot analysis detecting PPtase expression was further applied (Figure 4C). The GS115NpgA-CT and GS115-NpgA-SKL-CT-SKL clearly presented the PPtase band of about $40 \mathrm{KDa}$ while neither did GS115 nor GS115-3.5 K-CT-SKL. These results suggested that genes transcription and PPtase expression functioned well.

\section{Phosphopantetheinylation activity analysis of PPTase on ACP domain of PksCT}

To determine whether or not $A$. nidulans PPtase could react with $\mathrm{ACP}$ domain of $M$. purpureus $\mathrm{PksCT}$, an in vitro experiment was then studied. The PPTase containing 


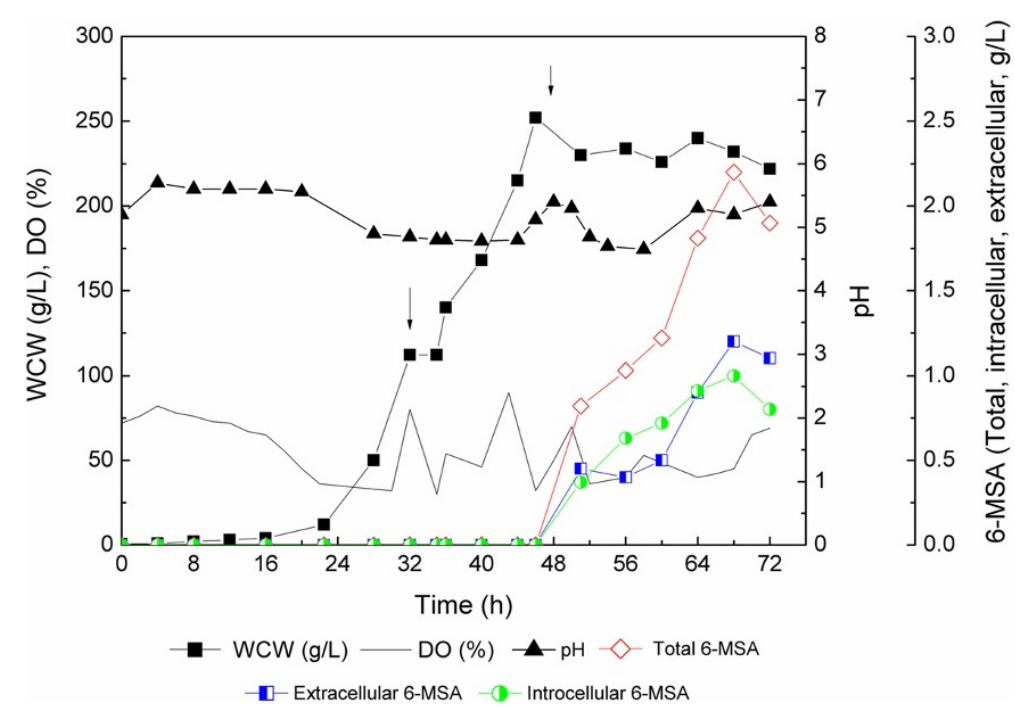

Figure 3 Time profiles of GS115-NpgA-ATX in 5-L stirred-tank bioreactor fermentation. The first arrow (32 h) indicated the starting point of glycerol feeding; The second arrow (47 h) indicated the starting point of methanol induction. Culture conditions were shown in Methods.

344 amino acids encoded by $A$. nidulans npgA (GenBank: AAF12814) was expressed by $P$. pastoris and purified with Nickel-affinity chromatography. The molecular weight $(\mathrm{Mw})$ of PPtase with His taq was calculated as $40 \mathrm{KDa}$ by the software Scansite $\mathrm{pI} / \mathrm{Mw}$. As shown in Figure 5B,

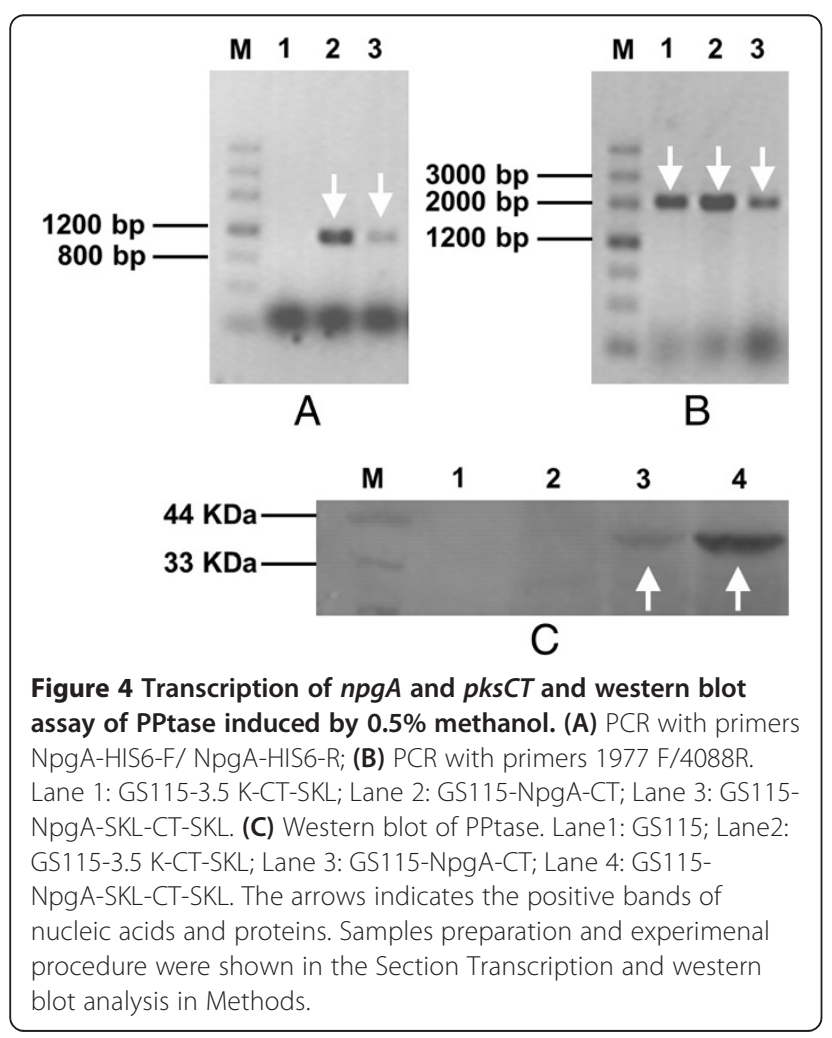

PPTase clearly exhibited in SDS-PAGE with a Mw about $40 \mathrm{Kda}$.

The ACP domain of fugnal PKS citrinin synthase (PksCT) from M. purpureus was employed to test the activity of PPtase. The ACP domain was analyzed and determined by Udwary-Merski algorithm (UMA) procedure (Figure 5A) [22,23] relying on the assumptions that amino acids in domain regions are relatively more conserved while linker regions carry more mutations and higher hydrophilicity [24]. E. coli BL21 was used for expression of ACP and its mutant ACPm (conserved serine at site 56 to alanine) (Figure 5A), and the proteins clearly appeared in the SDS-PAGE with similar $\mathrm{Mw}$ of about $20 \mathrm{Kda}$ (Figure 5C).

The bioactivity of PPtase was evaluated based on fluorescence labelled strategy. As we know, for function of PKS, the apo-ACP requires post-translational modification to form holo-ACP through the addition of phosphopantetheine derived from coenzyme A (CoA) to a conserved serine residue catalyzed by PPtase. If the conserved serine is mutated, the phosphopantetheinylation will then fail. Fluorescent probes Alexa Fluor 647C2maleimide and Bodipy FL N-(2-aminoethyl)maleimide can label phosphopantetheine by reacting with its hydrosulphonyl group. When fluorescently labelled 4'-phosphopantetheine linked with apo-ACP, the resulted holo-ACP would show fluorescence and stabilize in the SDS-PAGE [25]. However, once the conserved serine of ACP was mutated, it could not be labelled and emit fluorescence. In point of this study, once the PPtase encoded by $A$. nidulans npgA works, Alexa Fluor-647-phosphopantetheine labelled ACP would emit red fluorescence 


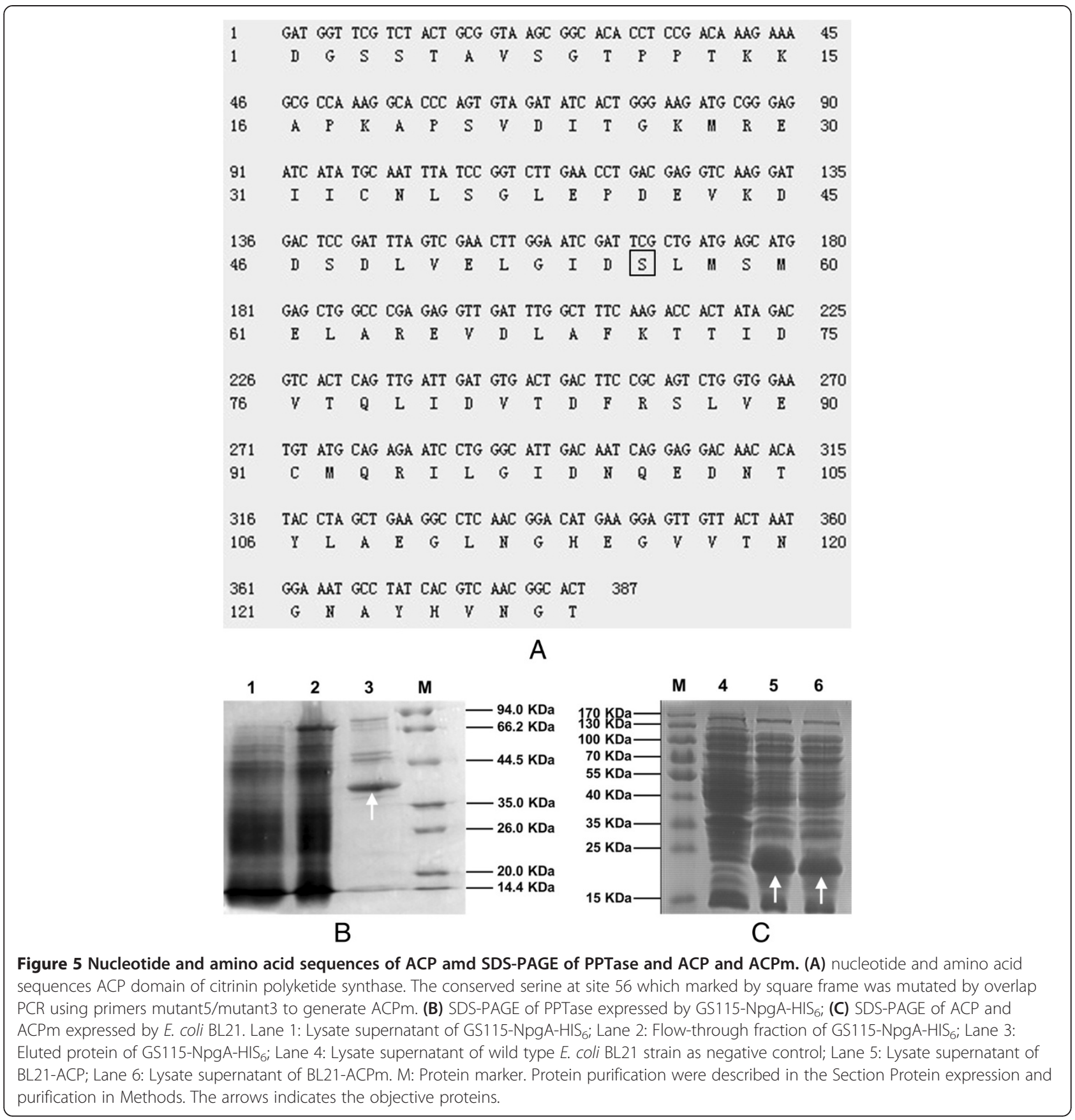

with $480 \mathrm{~nm}$ exciting light and $535 \mathrm{~nm}$ filter, while Bodipy FL-phosphopantetheine labelled ACP would emit green fluorescence with $580 \mathrm{~nm}$ exciting light and $670 \mathrm{~nm}$ filter.

As shown in Figure 6, Lane 1 and 3 were loaded with ACPm (conserved serine at site 56 to alanine) of PksCT that deactivating transthioesterification while Lane 2 and 4 contained the correct ACP of PksCT without mutation. Bodipy FL- phosphopantetheine labelled ACP in Lane 2 emitted green fluorescence when excited with wavelength of $480 \mathrm{~nm}$ (Figure 6A), while Alexa Fluor-
647- phosphopantetheine labelled ACP in Lane 4 produced red fluorescence when excited with wavelength of $580 \mathrm{~nm}$ (Figure 6B). However, Lane 1 and 3 emitted no fluorescence under different wavelengths of exciting lights and filters (Figure 6A\&B). The gel was then stained by Coomassie brilliant blue R250 solution and both ACP and ACPm presented correct Mw (Figure $6 \mathrm{C}$ ). The results proved that $A$. nidulans PPtase possessed good bioactivity for catalyzing modification of M. purpureus ACP. However, if the modification sites were mutated, i.e., ACPm, it lost ability of phosphopantetheinylation so that deprived 


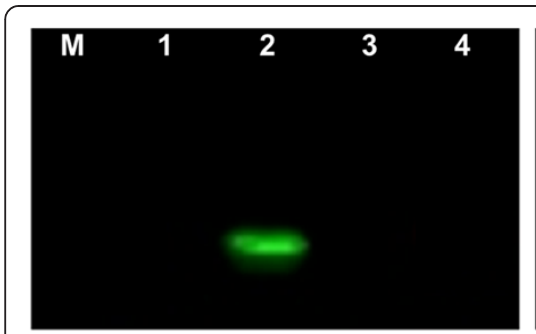

A

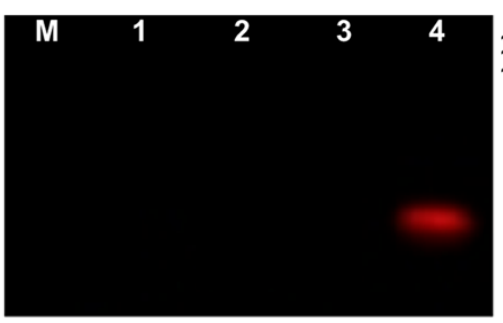

B

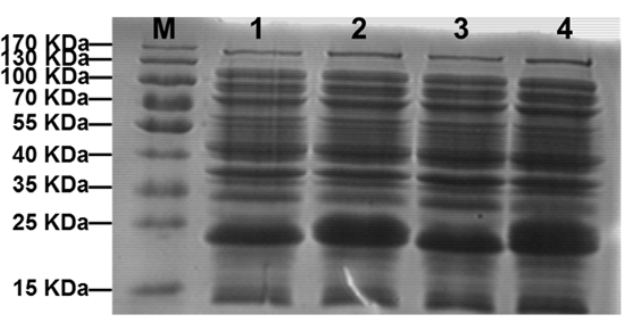

C

Figure 6 Fluorescent assay of ACP and ACPm that were fluorescently labelled. The KODAK In-vivo multispectral imaging systerm F was used for scanning. (A) Image parameter of $480 \mathrm{~nm}$ laser and $535 \mathrm{~nm}$ emission filter; (B) Image parameter of $580 \mathrm{~nm}$ laser and $670 \mathrm{~nm}$ emission filter; (C) Image of the same SDS-PAGE gel stained with Coomassie brilliant blue R-250. Lane 1: In vitro reaction containing Bodipy FL-CoA, PPtase and ACPm; Lane 2: In vitro reaction containing Bodipy-CoA, PPtase and ACP; Lane 3: In vitro reaction containing Alexa Fluor 647-CoA, PPtase and ACPm; Lane 4: In vitro reaction containing Alexa Fluor 647-CoA, PPtase and ACP. Protein preparation were described in Methods.

transthioesterification function for polyketide biosynthesis. This indicated that inefficient phosphopantetheinylation should not be the reason why citrinin intermediate was not observed. Other factors may affect polyketide synthesis that include activities of the individual catalytic domains and release of the product from the synthase [25]. The pksCT may also be poorly active without other proteins. Cox and coworkers reported that coexpression of different genes from fungal PKS clusters could lead to increased titres of objective compounds $[5,26]$. Coexpression of $\operatorname{ten} B$ with $\operatorname{ten} A$, ten $C$ and $\operatorname{ten} S$ with individual promoters and terminators increased Pretenellin-B by $149.2 \%$ in heterogolous expression in A. oryzae as compared to that without $\operatorname{ten} B$ [5]. Also, a hybrid fungal PKS-NRPS system TENS-PKS:DMBS-NRPS appeared to be more active than either of its progenitors and produced much higher purified yields of prototenellin C [26]. Additionally, some correct fungal polyketides may only be obtained by coexpression of their encoded PKS genes and other related genes. For instance, expression of tenS in the absence of a trans-acting ER component encoded by orf3 led to errors in assembly of the polyketide component, while coexpression of both genes worked correctly [27]. The lov $F$ encoding lovastatin nonaketide synthase only functioned accurately with coexpression with $\operatorname{lov} C$ encoding a trans-acting enoyl reductase in the heterologous production of monacolin-J [28]. Sakai et al. have recently reported a successful case of heterologous production of citrinin in $A$. oryzae by involving a small citrinin gene cluster (less than $20 \mathrm{~kb}$ ) containing $p k s C T$, activator gene $\operatorname{ctn} A$ and other 4 ORFs [16]. It is necessary for us to express this gene cluster or coexpression $p k s C T$ with related genes to further evaluate the $P$. pastoris system and probe into the source for this unsuccessful expression of $p k s C T$ gene.

Previous study have succeeded in heterologous production of fungal polyketides by involving A. nidulans PPtase, revealing that PPtase worked well on ACP of
6-MSAS and LNKS origining from $P$. patulum and A. terrus, respectively $[13,25]$. In this study, A. nidulans PPtase reacting with ACP of $M$. purpureus was also proven. These results indicated that $A$. nidulans PPtase probably work on ACPs from a wide range of filamentous fungi.

This work revealed that $P$. pastoris could be a good host that serve for heterogous expression of native polyketide or engineering recombinant polyketide biosynthesis in lab study. It also held great potential for heterologous production of polyketides in industrial application. We believed that $P$. pastoris could show more strong productive ability in heterologous production of non-lethal polyketides. Moreover, in view of the relative ease of performing genetic engineering the metabolic network modification may readily acheived to enhance precursor or intermediate formation or weaken its competing pathway for polyketide biosynthesis in P. pastoris. A successful case expanding the range of starter and extender units for polyketide biosynthesis has been illustrated in $S$. cerevisiae by introducing pathways for methylmalonyl-CoA generation [11]. However, the $P$. pastoris system may also faces the chellenges that other hosts ever encountered. The presence of introns and strain-specific splicing mechanisms for fungal PKS genes may bring hardness in verification of full length cDNA or cumbersome removal of introns [4]. The huge size of gene cluster encoding the biosynthetic pathway of polyketides may render expression constructs instable [4]. Also, some unknown origins caused blocks of compound synthesis, such as citrinin intermediate in this study and lovastatin precursors in S. cerevisiae [25]. Anyway, $P$. pastoris showed great potential to be a good host for polyketide production, and applying $P$. pastoris for a full PKS gene cluster for compound production would deeply test out its prospect in industrial application and becomes another research key point in the near future. 


\section{Conclusions}

An efficient $P$. pastoris expression system of fungal polyketides was constructed. The recombinant at $X$ strain successfully synthesized the objective compound 6-MSA and produced a high concentration $(2.2 \mathrm{~g} / \mathrm{L})$ of $6-\mathrm{MSA}$ in 5-L bioreactor fermentation. The $P$. pastoris expression system holds good potential for future industrial application of 6-MSA and other fungal polyketides, despite that its universality and the mystery of ineffectual cases still need further research.

\section{Methods}

\section{Molecular biology techniques}

Oligonucleotides were synthesized by Shanghai Generay Biotech Co., Ltd., China (Table 1). For PCR experiments, standard protocols were applied following PCR amplification kit (TaKaRa, cat. no. R011). Fungal genomic DNA was extracted by Plant Genomic DNA Extraction Kit (Tiangen Biotech Co., Ltd., China). E.coli vector pET28a was purchased from TaKaRa. P. pastoris GS115, E.coli TOP10, E.coli BL21 and yeast vectors pPICZ B and pPIC3.5 K were purchased from Invitrogen. A. nidulans A1149 (TN02A3) was bought from Fungal Genetics Stock Center, USA. Fluorescent probes Alexa Fluor 647C2maleimide and Bodipy FL N-(2-aminoethyl)maleimide were purchased from Invitrogen. DNase I was bought from Promega. Transformation of yeast cells and transformants screening were carried out according to Pichia protocols by Cregg and Russell [29]. Transformation and other standard recombinant DNA operations used in this study for $E$. coli were performed as described previously [30].

\section{Plasmids and strains}

The plasmids and strains used in this study were listed in Table 2.

\section{Strain GS115-NpgA , GS115-NpgA-ATX and GS115-ATX}

Using primers NpgAF/NpgAR1 to amplify $A$. nidulans $n p g A$, the obtained product was diluted 10 times and then used as DNA template to amplify $n p g A$ with $C$-terminal c-myc-HA taq by primers NpgAF/NpgAR2. The 1116 bp product was digested with SnaBI/NotI and ligated into vector pPIC3.5 K opened with SnaBI/NotI to yield the expression vector pPIC3.5 K-NpgA. After transforming into E. coli TOP 10, PCR verification by primers $5 \mathrm{AOX} 1 / 3 \mathrm{AOX} 1$ and sequencing, the correct plasmid was then linearized with SalI and transformed by electroporation into wild-type $P$. pastoris strain GS115 and the positive transformant GS115-NpgA was selected with histidine self-synthesis ability. The MSAS encoding gene $a t X$ was first amplified from the plasmid pESC-ATX recevied from Prof. Fujii [18]. The primers AtxF/AtxR were used and a DNA fragment of $5437 \mathrm{bp}$ was harvested. It was then digested by EcoRI/NotI and ligated into EcoRI/NotI sites of the opened vector pPICZ $\mathrm{B}$ to yield the expression plasmid pPICZ B-ATX. The plasmid was transformed into $E$. coli Top 10 , PCR verification by primers BstPF/AtxR and tested by sequencing. The correct plasmid was then amplified and digested by PmeI and then transformed by electroporation into $P$. pastoris GS115 and GS115-NpgA to form recombinant strains GS115-ATX and GS115-NpgA-ATX.

\section{Strain GS115-3.5 K, GS115-3.5 K-CT-SKL, GS115- NpgA-CT-SKL, GS115-NpgA-CT and GS115-NpgA-SKL-CT-SKL}

The commerial vector pPIC3.5 $\mathrm{K}$ acting as a control was transformed $P$. pastoris GS115 to gain strain GS115-3.5 K. A pksCT gene coding for citrinin synthase (PksCT) was first identified by reverse transcription to harvest cDNA [21]. Primer pairs CTAF/CTAR, CTBF/CTBR and CTCF/ CTCR were used to clone three fragments of $p k s C T$ and then they were digested and ligated into pPICZ B successively to produce a plasmid pPICZ B-CT-SKL having the complete $p k s C T$ gene with a SKL sequence for peroxisomal targeting. The plasmid was transformed into $E$. coli Top 10, PCR verification by primers CTAF/CTCR and tested by sequencing. The correct plasmid was then amplified and digested by PmeI and then transformed by electroporation into P. pastoris GS115-NpgA and GS115$3.5 \mathrm{~K}$ to form GS115-NpgA-CT-SKL and GS115-3.5 K-CT-SKL, respectively. Then, primers Citrinin-F2/ Citrinin-R2 were used for cloning a $p k s C T$ fragment from pPICZ B-CT-SKL and it was then digested by ClaI/NotI and ligated into pPICZ B-CT-SKL opened with ClaI/NotI to form pPICZ B-CT that removing the SKL sequence. The pPICZ B-CT transformed GS115-NpgA to get strain GS115-NpgA-CT. A pPIC3.5 K-NpgA-SKL plasmid was then constructed similar to pPIC3.5 K-NpgA, which only differed that involving a SKL sequence by primers NpgAF/NpgASR1 followed by NpgAF/NpgASR2 to direct NpgA into peroxisome. The pPIC3.5 K-NpgA-SKL transformed GS115 to generate GS115-NpgA-SKL, which was then transformed by pPICZ B-CT-SKL and produced GS115-NpgA-SKL-CT-SKL.

\section{Strain GS115-NpgA-HIS}

Genomic DNA of $A$. nidulans A1149 was first extracted. Primers NpgA-HIS 6 -F/NpgA-HIS 6 - $\mathrm{R}$ were designed and used for cloning npgA coding for PPTase from $A$. nidulans A1149 by involving His-tag and a PCR product of $1106 \mathrm{bp}$ was obtained. The product was digested with SacII/NotI and ligated into the same sites of the digested vector pPICZ $B$ to yield the expression vector pPICZ B-NpgA-HIS 6 . After transforming into E. coli TOP 10, PCR verification by primers $5 \mathrm{AOX} 1 / 3 \mathrm{AOX} 1$ and sequencing, the correct plasmid was then linearized with PmeI and transformed by electroporation into 
Table 1 Primers used in this study

\begin{tabular}{|c|c|}
\hline Primer & Sequence $\left(5^{\prime} \text { to } 3^{\prime}\right)^{a}$ \\
\hline \multirow[t]{2}{*}{ NpgA-HIS6-F } & GGACCGCGGCCACCATGGTGGACTACAAGGATGACGATGACAAGGGT \\
\hline & GGAGGTGGATCTGTGCAAGACACATCAAGCGCAAG \\
\hline NpgA-HIS6-R & ATAAGAATGCGGCCGCGCGGATAGGCAATTACACACC \\
\hline $5 \mathrm{AOX} 1$ & GACTGGTTCCAATTGACAAGC \\
\hline $3 \mathrm{AOX} 1$ & GCAAATGGCATTCTGACATCC \\
\hline AcpF & CATGGCTAGCGATGGTTCGTCTACTGCGGT \\
\hline AcpR & CCGCTCGAGTGCTGATTTTGGAGGATGGAACC \\
\hline Mutant5 & TGGAATCGATGCGCTGATGAGCATG \\
\hline Mutant3 & CATGCTCATCAGCGCATCGATTCCA \\
\hline NpgAF & GGAATTCTACGTAACCATGGTGCAAGACACATCAAGCGCAAG \\
\hline NpgAR1 & ATGGGTACAGATCCTCTTCTGAGATGAGTTITTGTTCGGATAGGCAATTACACACCCCAGTC \\
\hline NpgAR2 & ATAAGAATGCGGCCGCTTAAGCGTAATCTGGAACATCGTATGGGTACAGATCCTCTTCTGAGATG \\
\hline AtxF & CGGGAATTCACCATGGAGGTACATGGAGATGAAGTG \\
\hline AtxR & ATAAGAATGCGGCCGCCTTTCCCATCTITTCCAAAAACCAT \\
\hline BstpF & AACTGCAGAAGAATTCGGTTACCTGTACGTGGAAAAGGCTG \\
\hline BstpR & AACTGCAGAAGGTAACCCACCTTGGGAGGCGGCTG \\
\hline CTAF & TCCCCGCGGCATATGCAATTTATCCGGTCTTG \\
\hline CTAR & ATAAGAATGCGGCCGCTTACAACTTAGAATCTAGAAATCCCATGGTCTTCC \\
\hline CTBF & TCCCCGCGGTCGCGAGTACGAAGGTTATC \\
\hline CTBR & GGAATTCCATATGATCTCCCGCATCTTC \\
\hline CTCF & CGCCGCGGGACATGGCTCATCATCACCATCACCATGGTGGAGGTGGATCTATGATTGACTCAACTTCGCACTC \\
\hline CTCR & CTAGCCTCGCGATCACACCAAATACC \\
\hline Citrinin-F2 & CCATCGATTCGCTGATGAGCATGGAG-3 \\
\hline Citrinin-R2 & GGGAATTCGCGGCCGCATGATCTCTTGGCGCCCTG \\
\hline NpgASR1 & ATGGGTACAGATCCTCTTCTGAGATGAGTTITGTTCGGATAGGCAATTACACACCCCAGTC \\
\hline NpgASR2 & ATAAGAATGCGGCCGCTTACAACTTAGAAGCGTAATCTGGAACATCGTATGGGTACAGAT CCTCTTCTGAGATG \\
\hline $1977 \mathrm{~F}$ & TTCCTCAGCCCTACTGGTCAAT \\
\hline 4088R & GCTGGGATGCGTCTTGATAACC \\
\hline
\end{tabular}

${ }^{a}$ Restriction sites are underlined.

wild-type auxotrophic his4 strain P. pastoris GS115 to produce the recombinant strain GS115-NpgA-HIS 6 with zeocin resistence.

\section{Strains E. coli BL21-ACP and E. coli BL21-ACPm}

The UMA method was applied to identify the ACP domain of the citrinin synthase (PksCT) from $M$. purpureus [22]. Then primers AcpF/AcpR with NheI/ XhoI sites were designed based on the obtained DNA sequence. An ACP encoding DNA fragment of 534 bp was then cloned using primers AcpF/AcpR and cDNA from M. purpureus. The product was digested with NheI/XhoI and ligated into NheI/XhoI sites of the digested vector pET28a to yield the expression vector pET28a-ACP. The plasmid was transformed into E. coli Top 10, PCR verification by primers $5 \mathrm{AOX} 1 / 3 \mathrm{AOX} 1$ and tested by sequencing. The correct plasmid was then transformed into E. coli BL21 to yield recombinant strain BL21-ACP for protein expression.

As PPtase carries coenzyme A to the hydroxyl of conserved Ser residue in ACP to make it work, mutation at serine would inactivate it. Thus DNA fragment of ACPm were then constructed by over-lap PCR with primers mutant $5 /$ mutant 3 to change conserved serine at site 56 to alanine (Figure 5A). The strain BL21-ACPm was then constructed following the same procedure of strain pET28a-ACP.

\section{Medium and agent}

MGY medium: glycerol $10 \mathrm{~g} / \mathrm{L}$, YNB (Sigma) $6.7 \mathrm{~g} / \mathrm{L}$, sterilized at $121^{\circ} \mathrm{C}$ for $20 \mathrm{~min}$. Biotin was added to $0.4 \mathrm{mg} / \mathrm{L}$ after cooling. 
Table 2 List of plasmids and strains in this work

\begin{tabular}{|c|c|c|}
\hline Plasmids & Characteristic(s) & Reference \\
\hline pET28a $(+)$ & Kan $^{R} ;$ T7 promoter based E. coli expression vector & Novagen \\
\hline pET28a-ACP & pET28a(+) derivative carrying M. purpureus ACP encoded DNA & This study \\
\hline pET28a-ACPm & pET28a(+) derivative carrying M. purpureus ACPm encoded DNA & This study \\
\hline pESC-ATX & $\begin{array}{l}\text { Amp }{ }^{R} ; U R A 3 ; P_{G A L I} / G A L 10^{-b a s e d ~ y e a s t ~ e x p r e s s i o n ~ v e c t o r ; ~ c o m m e r i a l ~ p E S C-U R A ~} \\
\text { derivative carrying at } X \text { gene }\end{array}$ & Moriguchi et al., 2006 \\
\hline pPIC3.5 K & $A m p^{R}$ G418 $;$ HIS4; $P_{A O X 1}$-based yeast expression vector & Invitrogen \\
\hline pPICZ B & Zeocin ${ }^{R} ; P_{A O X I}$-based expression yeast vector & Invitrogen \\
\hline pPIC3.5 K-NpgA & pPIC3.5 K derivative carrying A. nidulans npgA gene & This study \\
\hline pPICZ B-NpgA-HIS 6 & pPICZ B derivative carrying A. nidulans $n p g A$ gene & This study \\
\hline pPICZ B-ATX & pPICZ B derivative carrying $A$. terreus at $X$ gene & This study \\
\hline pPICZ B-CT-SKL & pPICZ B derivative carrying M. purpureus pksCT gene a $P$. pastoris SKL sequence & This study \\
\hline pPICZ B-CT & pPICZ B derivative carrying M. purpureus pksCT gene & This study \\
\hline pPIC3.5 K-NpgA-SKL & pPIC3.5 K derivative carrying A. nidulans $n p g A$ gene and a $P$. pastoris SKL sequence & This study \\
\hline Strains & Characteristic(s) & Reference \\
\hline GS115 & his4, AOX 1, AOX2 & Invitrogen \\
\hline GS115-NpgA-HIS 6 & Zeocin $^{\text {R}}$; GS115 with plasmid pPICZ B-NpgA-HIS 6 & This study \\
\hline GS115-ATX & Zeocin $^{R}$; GS115 with plasmid pPICZ B-ATX & This study \\
\hline GS115-NpgA & 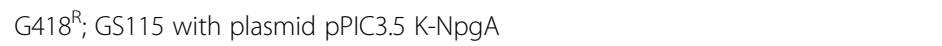 & This study \\
\hline GS115-NpgA-ATX & Zeocin $^{R}$; G418 ${ }^{R}$; GS115-NpgA with plasmid pPICZ B-ATX & This study \\
\hline GS115-3.5 K & G418 ${ }^{\mathrm{R}}$; GS115 with pPIC3.5 K & This study \\
\hline GS115-3.5 K-CT-SKL & Zeocin $^{\text {R }}$ G418 ; GS115-3.5 K with pPICZ B-CT-SKL & This study \\
\hline GS115-NpgA-CT & Zeocin $^{R}$; G418 ${ }^{R}$; GS115-NpgA with plasmid pPICZ B-CT & This study \\
\hline GS115-NpgA-SKL & G418 ${ }^{R}$; GS115 with plasmid pPIC3.5 K-NpgA-SKL & This study \\
\hline GS115-NpgA-CT-SKL & Zeocin $^{R}$; G418 ${ }^{R}$; GS115-NpgA with plasmid pPICZ B-CT-SKL & This study \\
\hline GS115-NpgA-SKL-CT-SKL & Zeocin $^{R}$; G418 ; GS115-NpgA-SKL with plasmid pPICZ B-CT-SKL & This study \\
\hline E. coli TOP10 & $\mathrm{F}^{\prime}$ [laclq, Tn10(TetR)] mcrA p80lacZ $\Delta \mathrm{M} 15 \Delta / a c X 74$ deoR recA1 & Invitrogen \\
\hline E. coli BL21 & $\mathrm{F}^{-}$ompT hsdS $\mathrm{S}_{B}^{-}\left(\mathrm{r}_{\mathrm{B}}^{-} \mathrm{m}_{\mathrm{B}}^{-}\right) \mathrm{gal} \mathrm{dcm}(\mathrm{DE} 3)$ & Invitrogen \\
\hline BL21-ACP & Kan ${ }^{R} ;$ BL21 with plasmid pET28a-ACP & This study \\
\hline BL21-ACPm & $\mathrm{Kan}^{\mathrm{R}}$; BL21 with plasmid pET28a-ACPm & This study \\
\hline
\end{tabular}

MM medium: YNB (Sigma) $13.4 \mathrm{~g} / \mathrm{L}$, sterilized at $121^{\circ} \mathrm{C}$ for $20 \mathrm{~min}$. Biotin was added to $0.4 \mathrm{mg} / \mathrm{L}$ and methanol was added to $0.5 \%(\mathrm{v} / \mathrm{v})$ after cooling.

BSM medium: 85\% (w/v) $\mathrm{H}_{3} \mathrm{PO}_{4} 13 \mathrm{~mL} / \mathrm{L}, \mathrm{KOH}$ $10.6 \mathrm{~g} / \mathrm{L}, \mathrm{CaSO}_{4} 0.82 \mathrm{~g} / \mathrm{L}, \mathrm{K}_{2} \mathrm{SO}_{4} 18.2 \mathrm{~g} / \mathrm{L}, \mathrm{MgSO}_{4} \cdot 7 \mathrm{H}_{2} \mathrm{O}$ $14.9 \mathrm{~g} / \mathrm{L}$, glycerol $40 \mathrm{~g} / \mathrm{L},\left(\mathrm{NH}_{4}\right)_{2} \mathrm{SO}_{4} 13.2 \mathrm{~g} / \mathrm{L}$, Antifoam 204 (Sigma) $0.33 \mathrm{~mL} / \mathrm{L}$, sterilized at $121^{\circ} \mathrm{C}$ for $30 \mathrm{~min}$. PTM1 solution was then added with $4.4 \mathrm{~mL} / \mathrm{L}$ after cooling.

PTM1 solution: $\mathrm{CuSO}_{4} \cdot 5 \mathrm{H}_{2} \mathrm{O} 6.0 \mathrm{~g} / \mathrm{L}, \mathrm{NaI} 0.08 \mathrm{~g} / \mathrm{L}$, $\mathrm{MnSO}_{4} \cdot \mathrm{H}_{2} \mathrm{O} 3.0 \mathrm{~g} / \mathrm{L}, \mathrm{Na}_{2} \mathrm{MoO}_{4} \cdot 2 \mathrm{H}_{2} \mathrm{O} 0.2 \mathrm{~g} / \mathrm{L}, \mathrm{HBO}_{3}$ $0.02 \mathrm{~g} / \mathrm{L}, \mathrm{CoCl}_{2} \cdot 6 \mathrm{H}_{2} \mathrm{O} 0.914 \mathrm{~g} / \mathrm{L}, \mathrm{ZnCl}_{2} 20.0 \mathrm{~g} / \mathrm{L}$, $\mathrm{FeSO}_{4} \cdot 7 \mathrm{H}_{2} \mathrm{O} 65.0 \mathrm{~g} / \mathrm{L}$, biotin $0.2 \mathrm{~g} / \mathrm{L}, \mathrm{H}_{2} \mathrm{SO}_{4} 5.0 \mathrm{~mL} / \mathrm{L}$, sterilized by filtration with $0.22 \mu \mathrm{m}$ membrane filter and stored at $4^{\circ} \mathrm{C}$ away from light.
Glycerol feeding medium: glycerol 50\% (w/v), sterilized at $121^{\circ} \mathrm{C}$ for $30 \mathrm{~min}$. PTM1 solution was then added with $12 \mathrm{~mL} / \mathrm{L}$ after cooling.

Methanol feeding medium: methanol (100\%) with 12 mL/L PTM1.

SDS-PAGE electrophoresis buffer: 1 g SDS, 3.03 g Tris, 14.4 $\mathrm{g}$ Glycine, dissolved in $1 \mathrm{~L}$ ultrapure water.

Coomassie brilliant blue R250 solution: $1.0 \mathrm{~g}$ Coomassie brilliant blue R250, $100 \mathrm{~mL}$ acetic acid, $300 \mathrm{~mL}$ ethanol, dissolved in $1 \mathrm{~L}$ ultrapure water.

Electrophoretic destain solution: $32 \mathrm{~mL}$ acetic acid, $100 \mathrm{~mL}$ ethanol, ultrapure water added to $1 \mathrm{~L}$.

SDS-PAGE loading buffer (5×): $1 \mathrm{~g}$ SDS, $2.5 \mathrm{~mL} 1 \mathrm{M}$ Tris- $\mathrm{HCl}(\mathrm{pH}$ 6.8), $5 \mathrm{~mL}$ glycerol, $0.05 \mathrm{~g}$ Coomassie brilliant blue, ultrapure water added to $10 \mathrm{~mL}$. 
Nickel-affinity chromatography binding buffer: $10 \mathrm{mmol} / \mathrm{L}$ $\mathrm{NaH}_{2} \mathrm{PO}_{4}, 10 \mathrm{mmol} / \mathrm{L} \quad \mathrm{Na}_{2} \mathrm{HPO}_{4}, 0.5 \mathrm{~mol} / \mathrm{L} \mathrm{NaCl}$, $20 \mathrm{mmol} / \mathrm{L}$ imidazole, $\mathrm{pH}$ 7.4.

Nickel-affinity chromatography elution buffer: $10 \mathrm{mmol} / \mathrm{L}$ $\mathrm{NaH}_{2} \mathrm{PO}_{4}, \quad 10 \mathrm{mmol} / \mathrm{L} \quad \mathrm{Na}_{2} \mathrm{HPO}_{4}, \quad 0.5 \mathrm{~mol} / \mathrm{L} \quad \mathrm{NaCl}$, $500 \mathrm{mmol} / \mathrm{L}$ imidazole, $\mathrm{pH}$ 7.4.

Fluorescent labeling reaction buffer: $137 \mathrm{mM} \mathrm{NaCl}$, $10 \mathrm{mM}$ PBS (pH 7.4), $50 \mathrm{mM} \mathrm{MgCl}_{2}$.

CoA reaction buffer:75 mM Tris- $\mathrm{HCl}$ (pH 8.8), $10 \mathrm{mM} \mathrm{MgCl}_{2}, 25 \mathrm{mM}$ DTT.

$20 \mathrm{mM}$ Tris dialysis buffer: $20 \mathrm{mM}$ Tris- $\mathrm{HCl}(\mathrm{pH} 7.5)$, $150 \mathrm{mM} \mathrm{NaCl}$.

\section{Culture conditions}

For GS115-NpgA-HIS 6 , a singal colony was inoculated into a $250 \mathrm{~mL}$ shake flask containing $25 \mathrm{~mL}$ YPD medium and cultivated at $30^{\circ} \mathrm{C}$ and $200 \mathrm{rpm}$ for about 16 hours. Afterwards, the broth was transferred into $25 \mathrm{~mL}$ MGY medium to make the $\mathrm{OD}_{600}$ about 0.03 . The liquid was centrifugated at $3000 \mathrm{~g}$ for $5 \mathrm{~min}$, washed with MM medium twice and suspended with MM medium to be $\mathrm{OD}_{600}=1.0$. Then $50 \mathrm{~mL}$ liquid was then transferred into $500 \mathrm{~mL}$ baffled shake flask, covered two layers of gauze and cultivated at $30^{\circ} \mathrm{C}$ and $200 \mathrm{rpm}$. Methanol was fed every 24 hours to keep its concentration around $0.5 \%$ to induce protein expression.

For BL21-ACP and BL21-ACPm, a singal colony was inoculated into a $250 \mathrm{~mL}$ shake flask containing $25 \mathrm{~mL}$ LB medium with $50 \mu \mathrm{g} / \mathrm{mL}$ kanamycin and incubated at $37^{\circ} \mathrm{C}$ and $200 \mathrm{rpm}$ for 12 hours. Then it was inoculated into $25 \mathrm{~mL} \mathrm{LB}$ medium with $50 \mu \mathrm{g} / \mathrm{mL}$ kanamycin in $250 \mathrm{~mL}$ shake flask by $1 \%(\mathrm{v} / \mathrm{v})$ and cultivated at $37^{\circ} \mathrm{C}$ and $200 \mathrm{rpm}$ until $\mathrm{OD}_{600}=0.6$. Afterwards, $0.5 \mathrm{mM}$ IPTG was added to induce protein expression for 5 hours. The broth was centrifugated at $8000 \mathrm{~g}$ for $3 \mathrm{~min}$, washed with binding buffer and then stored at $-20^{\circ} \mathrm{C}$ for forward analysis.

The shake flask cultures of GS115-ATX, GS115NpgA-ATX, GS115- NpgA-CT-SKL, GS115-3.5 K-CTSKL, GS115-NpgA-CT and GS115-NpgA-SKL-CT-SKL were as same as that of GS115-NpgA-HIS 6 . For 5-L bioreactor, $300 \mathrm{~mL}$ seeds $\left(\mathrm{OD}_{600}=6.0\right)$ cultivated with MGY medium were collected and inoculated into a 5-L stirred-tank bioreactor (Shanghai Guoqiang Bioengineering Equipment Co., Ltd.) containing 3 L BSM medium. The impeller equipped was double layer sixblade Rushton disc turbine (RDT, $6.8 \mathrm{~cm}$ i.d.). The lower impeller was $2.5 \mathrm{~cm}$ above the reactor bottom, and the vertical distance between two impellers was $7.2 \mathrm{~cm}$. Dissolved oxygen (DO) was measured using a polarographic probe calibrated to $100 \%$ saturation for aeration of $1 \mathrm{vvm}$ at agitation of $600 \mathrm{rpm}$ and tank inside pressure of $0.02 \mathrm{Mpa}$. The broth $\mathrm{pH}$ was controlled at 5.0 by $\mathrm{NH}_{4} \mathrm{OH}$. The temperature was kept at $30^{\circ} \mathrm{C}$ and the
DO was controlled over $30 \%$ by adjusting agitation (not higher than $800 \mathrm{rpm}$ ) and aeration (mixed gas of oxygen and air if needed). When glycerol used up and DO repidly increased, glycerol feeding medium was limitedly fed by $8 \mathrm{~mL} / \mathrm{L} / \mathrm{h}$ for 2 hours. The feeding rate may accelerate but keep DO not lower than 30\% until the wet cell weight (WCW) reached $250 \mathrm{~g} / \mathrm{L}$. After glycerol was exhausted, methanol feeding medium were fed by $4 \mathrm{~mL} / \mathrm{h} / \mathrm{L}$ for 2 hours and then slowly increased to $12 \mathrm{~mL} / \mathrm{h} / \mathrm{L}$ after 6 more hours and keep this rate until the end.

\section{Protein expression and purification}

To prepare PPtase, $30 \mathrm{OD}_{600}$ units of GS115-NpgA-HIS cells were harvested by centrifugation at $6,000 \mathrm{~g}$ for $3 \mathrm{~min}$, washed twice with ice-cold $50 \mathrm{mM}$ binding buffer ( $\mathrm{pH} 7.4$ ), and then frozen at $-20^{\circ} \mathrm{C}$. Cells were thawed and resuspended in $1 \mathrm{~mL}$ binding buffer ( $\mathrm{pH}$ 7.4). It were mixed with $1.8 \mathrm{~g}$ glass beads (Biospec Products, Bartlesville, OK) in a $2.0-\mathrm{mL}$ screw-cap tube followed by disruption with a bead disrupter (MiniBeadBeater-8; Biospec Products) for 8 cycles (1 min vibrating and $1 \mathrm{~min}$ resting in ice for each cycle). The lysate was centrifuged at $12,000 \mathrm{~g}$ for $30 \mathrm{~min}$ and the solid was discarded. It was then filtrated by $0.22 \mu \mathrm{m}$ membrane and loading to the column that balanced well with binding buffer by $0.5 \mathrm{~mL} / \mathrm{min}$. Afterwards, the column was washed with binding buffer of 10 column volumes to discard other proteins, followed by elution buffer of 5 column volumes to collect the proteins. The eluent was dialyzed by $20 \mathrm{mM}$ Tris- $\mathrm{HCl}$ (pH 7.5) twice and concentrated to $4 \mathrm{mg} / \mathrm{mL}$ by ultrafiltration, and then used for in vitro experiment.

Cells of BL21-ACP and BL21-ACPm were centrifugated and resuspended with $20 \mathrm{mM}$ Tris- $\mathrm{HCl}$ ( $\mathrm{pH}$ 7.5). The proteins were then extracted by ultrasonic disruption ( $300 \mathrm{w}, 3 \mathrm{~s}$ on, $4 \mathrm{~s}$ off, $15 \mathrm{~min}$ ) and collected by centrifugation at $12000 \mathrm{~g}$ and $4^{\circ} \mathrm{C}$ for $20-30 \mathrm{~min}$ (supernatant).

Eluted samples were analyzed on a Tris-Gly SDSPAGE gel (Invitrogen) and stained with Coomassie brilliant blue R250 solution.

\section{Fluorescence labeling of $\mathrm{CoA}$ and bioactivity analysis of PPTase}

Preparation of Alexa Fluor 647-CoA: Dissolve $0.02 \mathrm{mg}$ Alexa Fluor 647-C2maleimide in $20 \mu \mathrm{L} 50 \%$ DMSO. It was then mixed with $1.0 \mathrm{mg} \mathrm{CoA}(0.53 \mu \mathrm{M})$ and $30 \%$ DMSO (v/v), fed with fluorescent labeling reaction buffer up to $245 \mu \mathrm{L}$, and reacted in dark for 1 hour. The Alexa Fluor 647-CoA was then purified referring to previous study [25].

Preparation of Bodipy FL-CoA: Dissolve $1 \mathrm{mg}$ bodipy FL N-(2-aminoethyl)maleimide $40 \mu \mathrm{L}$ DMSO. It was then mixed with $0.8 \mathrm{mg} \mathrm{CoA}$ and 10\% DMSO (v/v), fed with fluorescent labeling reaction buffer up to $2 \mathrm{~mL}$ and 
reacted in dark for $85 \mathrm{~min}$. The liquor was then extracted with ethyl acetate for three times and the aqueous phase with Bodipy FL-CoA was collected.

The $25 \mu \mathrm{L}$ reaction system consisted of $9.5 \mu \mathrm{M}$ Alexa Fluor 647-CoA (or $2 \mu \mathrm{L}$ Bodipy-CoA), $0.16 \mu \mathrm{g} / \mu \mathrm{L}$ purified His6-NpgA (PPtase), $5 \mu \mathrm{L}(200 \mu \mathrm{g})$ protein supernant of BL21-ACP(or BL21-ACPm) in CoA reaction buffer. After incubated at room temperature for $10 \mathrm{~min}$, loading buffer was added to terminate the reaction and used for SDSPAGE after heated at $100^{\circ} \mathrm{C}$ for $2 \mathrm{~min}$.

The KODAK In-Vivo multispectral imaging system was used for the gel analysis by setting different wave length of exciting light. After analysis, the gel was photoed under white light after stained by Coomassie brilliant blue R250 solution and destained in electrophoretic destain solution.

\section{Extraction and identification of 6-MSA and citrinin intermediate}

For 6-MSA, $10 \mathrm{~mL}$ culture broth was centrifugated at $12000 \mathrm{~g}$ for $3 \mathrm{~min}$. The supernatant was fully extracted with $20 \mathrm{~mL}$ ethyl acetate, and the organic phase was distilled under reduced pressure and dissolved in $10 \mathrm{~mL}$ methanol. It was used for HPLC analysis after filtration and dilution. The cells was resuspended in $1 \mathrm{~mL}$ methanol and disrupted by bead disrupter just as described before. The mixture was then centrifugated at $12000 \mathrm{~g}$ for $3 \mathrm{~min}$ and the obtained supernant was filtrated and diluted before HPLC analysis.

The sample was analyzed by a HPLC system of Agilent 1100 with a C18 column (Kromasil ${ }^{\mathrm{T}}$, Sweden, $250 \mathrm{~mm} \times$ $4.6 \mathrm{~mm} \times 5 \mu \mathrm{m}, 100 \AA$-spherical silica). It was eluted with a gradiant strategy that $90 \%(\mathrm{v} / \mathrm{v}) \mathrm{A}($ water $+1 \% \mathrm{AcOH})$ and $10 \%(\mathrm{v} / \mathrm{v}) \mathrm{B}(\mathrm{CH} 3 \mathrm{CN}+1 \% \mathrm{AcOH})$ to $20 \%(\mathrm{v} / \mathrm{v}) \mathrm{A}$ and $80 \%$ $(\mathrm{v} / \mathrm{v}) \mathrm{B}$ in $30 \mathrm{~min}$, then to $100 \%(\mathrm{v} / \mathrm{v}) \mathrm{B}$ in $2 \mathrm{~min}$ and kept for $5 \mathrm{~min}$. Afterwards, it was then gradiantly changed to $90 \%(\mathrm{v} / \mathrm{v}) \mathrm{A}$ and $10 \%(\mathrm{v} / \mathrm{v}) \mathrm{B}$ in $3 \mathrm{~min}$. The elution conditions were set as that flow rate of $1.0 \mathrm{~mL} / \mathrm{min}$, UV absorbency of $306 \mathrm{~nm}$ and operating temperature of $30^{\circ} \mathrm{C}$. The compound was further confirmed by mass spectrometry (EI-MS, Agilent G2577A), and also nuclear magnetic resonance (NMR, BRUKER-400) with freeze-dried sample dissolved in deuterated DMSO for ${ }^{1} \mathrm{HNMR}$ analysis. For quantifying the production of 6-MSA, the standard 6-MSA (Shanghai Bepharm Co. Ltd) was used for standard curve preparation by the same HPLC procedure. The extracted 6-MSA samples from fermentation broth were then diluted properly for HPLC analysis and the 6-MSA production in fermentation broth was then calculated. The compound was also confirmed by EI-MS and ${ }^{1}$ HNMR analysis.

Extraction of citrinin intermediate was as same as 6-MSA. The HPLC system used was also the same. It was eluted with a gradiant strategy that $90 \%(\mathrm{v} / \mathrm{v})$ A $($ water $+1 \% \quad \mathrm{AcOH})$ and $10 \% \quad(\mathrm{v} / \mathrm{v})$ B $(\mathrm{CH} 3 \mathrm{CN}+1 \%$ $\mathrm{AcOH})$ to $30 \%(\mathrm{v} / \mathrm{v}) \mathrm{A}$ and $70 \%(\mathrm{v} / \mathrm{v}) \mathrm{B}$ in $25 \mathrm{~min}$, then immediately change to $100 \%(\mathrm{v} / \mathrm{v}) \mathrm{B}$ and kept for $5 \mathrm{~min}$. The elution conditions were set as that flow rate of $1.0 \mathrm{~mL} / \mathrm{min}$, UV absorbency of wide wavelength range and operating temperature of $30^{\circ} \mathrm{C}$.

\section{Transcription and western blot analysis}

Total RNAs were prepared by RiboPure ${ }^{\mathrm{rm}}$-Yeast Kit (Ambion) following its standard protocol and were subjected to DNase I treatment to exclude the genomic DNA contaminant. Reverse transcription was performed following ReverTra Ace transcription kit (Toyobo). For western blot, samples were first prepared as same as that described in the Section of Protein expression and purification. Total proteins were separated on polyacrylamide gels under denaturing conditions [31] and then transferred onto a polyvinylidene difluoride (PVDF) membrane using the electrophoretic transfer method with rabbit anti-HA antibody (Tiangen) as the primary antibody and peroxidase-conjugated goat anti-rabbit immunoglobulin G (Jackson ImmunoResearch) as the secondary antibody.

\section{Additional file}

Additional file 1: Figure S1. Time profiles of $P$. pastoris GS115 in 5-L stirred-tank bioreactor fermentation with 6-MSA feeding. The first arrow (24 h) indicated the starting point of glycerol feeding; The second arrow (32 h) indicated the time point of 6-MSA feeding.

\section{Competing interests}

The authors declare that they have no competing interests.

\section{Authors' contributions}

LG and MC designed the experiments, LG conducted most of the experiments. LG, WS and SX performed bioreactor fermentations. $L G$ and $M C$ analyzed the results. $M C$ wrote the manuscript. $X Z$ and $Y Z$ reviewed and revised the manuscript. All authors read and approved the final manuscript.

\section{Acknowledgement}

This work was supported by National High Technology Research and Development Program of China (2011AA090702, 2012AA092103, 2012AA092105) and National Natural Science Funds of China (31270141,41306121). We thank Prof. Fujii for kind supply of plasmid PESC-ATX.

Received: 28 July 2013 Accepted: 4 September 2013

Published: 8 September 2013

\section{References}

1. Cox RJ: Polyketides, proteins and genes in fungi: programmed nano-machines begin to reveal their secrets. Org Biomol Chem 2007, 5:2010-2026.

2. Weissman KJ, Leadlay PF: Combinatorial biosynthesis of reduced polyketides. Nat rev microbiol 2005, 3:925-936.

3. Wattanachaisaereekul S, Lantz AE, Nielsen ML, Andrésson ÓS, Nielsen J: Optimization of heterologous production of the polyketide 6-MSA in Saccharomyces cerevisiae. Biotechnol Bioeng 2007, 97:893-900.

4. Schümann J, Hertwerk C: Advances in cloning, functional analysis and heterologous expression of fungal polyketide synthase genes. J Biotechnol 2006, 124:690-703.

5. Heneghan MN, Yakasai AA, Halo LM, Song ZS, Bailey AM, Simpson TJ, Cox RJ, Lazarus CM: First heterologous reconstruction of a complete 
functional fungal biosynthetic multigene cluster. Chem Bio Chem 2010, 11:1508-1512

6. Macauley-Patrick S, Fazenda ML, McNeil B, Harvey LM: Heterologous protein production using the Pichia pastoris expression system. Yeast 2005, 22:249-270.

7. Cereghino JL, Cregg JM: Heterologous protein expression in the methylotrophic yeast Pichia pastoris. FEMS Microbiol Rev 2000, 24:45-66.

8. Dimroth $P$, Ringelmann $E$, Lynen F: 6-Methylsalicylic acid synthetase from Penicillium patulum. Some catalytic properties of the enzyme and its relation to fatty acid synthetase. Eur J Biochem 1976, 68:591-596.

9. Beck J, Ripka S, Siegner A, Schiltz E, Schweizer E: The multifunctional 6-methylsalicylic acid synthase gene of Penicillium patulum. Its gene structure relative to that of other polyketide synthases. Eur J Biochem 1990, 192:487-498.

10. Fujii I, Ono Y, Tada H, Gomi K, Ebizuka Y, Sankawa U: Cloning of the polyketide synthase gene atX from Aspergillus terreus and its identification as the 6-methylsalicylic acid synthase gene by heterologous expression. Mol Gen Genet 1996, 253:1-10.

11. Mutka SC, Bondi SM, Carney JR, Da Silva NA, Kealey JM: Metabolic pathway engineering for complex polyketide biosynthesis in Saccharomyces cerevisiae. FEMS Yeast Res 2006, 6:40-47.

12. Bedford DJ, Schweizer E, Hopwood DA, Khosla C: Expression of a functional polyketide synthase in the bacterium Streptomyces coelicolor A3(2). J Bacterio/ 1995, 177:4544-4548.

13. Kealey JT, Liu L, Santi DV, Betlach MC, Barr PJ: Production of a polyketide natural product in nonpolyketide-producing prokaryotic and eukaryotic hosts. Proc Natl Acad Sci 1998, 95:505-509.

14. Yalpani N, Altier DJ, Barbour E, Cigan AL, Scelonge CJ: Production of 6-methylsalicylic acid by expression of a fungal polyketide synthase activates disease resistance in tobacco. Plant Cell 2001, 13:1401-1409.

15. Abou-Zeid AM: Review on citrinin: production, effect of some plant extracts and gene involved in its biosynthesis. J Civil Environment Engg 2012, 2:1000113.

16. Sakai K, Kinoshita H, Shimizu T, Nihira T: Construction of a citrinin gene cluster expression system in heterologous Aspergillus oryzae. J Biosci Bioeng 2008, 106:466-472.

17. Hajjaj H, Klaébé A, Loret MO, Goma G, Blanc PJ, Francois J: Biosynthetic pathway of citrinin in the filamentous fungus monascus ruber as revealed by ${ }^{13} \mathrm{C}$ nuclear magnetic resonance. Appl Environ Microbiol 1999, 65:311-314

18. Moriguchi T, Ebizuka Y, Fujii I: Analysis of subunit interactions in the iterative type I polyketide synthase ATX from Aspergillus terreus. Chembiochem 2006, 7:1869-1874.

19. Khadem S, Marles RJ: Monocyclic phenolic acids; hydroxy- and polyhydroxybenzoic acids: occurrence and recent bioactivity studies. Molecules 2010, 15:7985-8005.

20. Wattanachaisaereekul S, Lantz AE, Nielsen ML, Nielsen J: Production of the polyketide 6-MSA in yeast engineered for increased malonyl-CoA supply. Metab Eng 2008, 10:246-254.

21. Shimizu T, Kinoshita H, Ishihara S, Sakai K, Nagai S, Nihira T: Polyketide Synthase Gene Responsible for Citrinin Biosynthesis in Monascus purpureus. Appl Environ Microbiol 2005, 71:3453-3457.

22. Udwary DW, Merski M, Townsend CA: A method for prediction of the locations of linker regions within large multifunctional proteins, and application to a type I polyketide synthase. J Mol Biol 2002, 323:585-598.

23. Wallqvist A, Fukunishi Y, Murphy LR, Fadel A, Levy RM: Iterative sequence/ secondary structure search for protein homologs: comparison with amino acid sequence alignments and application to fold recognition in genome databases. Bioinformatics 2000, 16:988-1002.

24. Kong L, Ranganathan S: Delineation of modular proteins: domain boundary prediction from sequence information. Brief Bioinform 2004, 5:179-192.

25. Lee KKM, Da Silva NA, Kealey JT: Determination of the extent of phosphopantetheinylation of polyketide synthases expressed in Escherichia coli and Saccharomyces cerevisiae. Ana Biochem 2009, 394:75-80.

26. Heneghan MN, Yakasai AA, Williams K, Kadir KA, Wasil Z, Bakeer W, Fisch KM, Bailey AM, Simpson TJ, Cox RJ, Lazarus CM: The programming role of trans-acting enoyl reductases during the biosynthesis of highly reduced fungal polyketides. Chem Sci 2011, 2:972-980.
27. Halo LM, Marshall JW, Yakasai AA, Song Z, Butts CP, Crump MP, Heneghan M, Bailey AM, Simpson TJ, Lazarus CM, Cox Rl: Authentic heterologous expression of the tenellin iterative polyketide synthase nonribosomal peptide synthetase requires coexpression with an enoyl reductase. ChemBioChem 2008, 9:585-594.

28. Kennedy J, Auclair K, Kendrew SG, Park C, Vederas JC, Hutchinson CR: Modulation of polyketide synthase activity by accessory proteins during lovastatin biosynthesis. Science 1999, 284:1368-1372.

29. Cregg JM, Russell KA: Transformation. In Pichia protocols, Volume 103. Edited by Higgins DR, Cregg JM. Totowa: Humana Press; 1998:27-39.

30. Sambrook J, Russell DW: Molecular Cloning: A Laboratory Manual. 3rd edition. New York: Cold Spring Harbor Laboratory Press; 2001

31. Wang $Y, X$, $Y$ n $Y$, Zhang $P$, Jiang $X$, Ni Z, Tong $L$, Zhou $X$, Lin $L$, Ding J, Zhang $Y$ : Targeting expression of the catalytic domain of kinase insert domain receptor (KDR) in the peroxisomes of Pichia pastoris. FEMS Yeast Res 2009, 9:732-741.

doi:10.1186/1475-2859-12-77

Cite this article as: Gao et al:: Engineered fungal polyketide biosynthesis in Pichia pastoris: a potential excellent host for polyketide production. Microbial Cell Factories 2013 12:77.

\section{Submit your next manuscript to BioMed Central and take full advantage of:}

- Convenient online submission

- Thorough peer review

- No space constraints or color figure charges

- Immediate publication on acceptance

- Inclusion in PubMed, CAS, Scopus and Google Scholar

- Research which is freely available for redistribution 